\title{
SELECTION OF RICE VARIETIES SUITABLE FOR RICE BASED FOOD PRODUCTS
}

\author{
By \\ Mohamed Jaleel Mohamed Fari
}

Thesis submitted to the University of Sri Jayewardenepura for the award of the

Degree of Master of Philosophy in Food Science and Technology

on

September 2009. 
The work described in this thesis was carried out by me under the supervision of Mrs. Damitha Rajapaksa and Prof. K.K.D.S Ranaweera and a report on this has not been submitted in whole or in part to any University or any other institution for another Degree/Diploma.

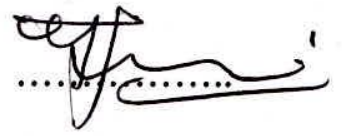

M.J.M. Fari

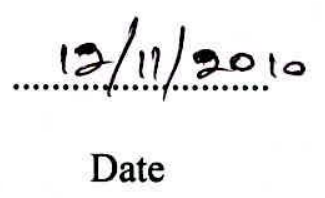

Date 
We certify that the above statement made by the candidate is true and that this thesis is suitable for submission to the University for the purpose of evaluation.

D. Rayapakea

Mrs. Damitha Rajapaksa

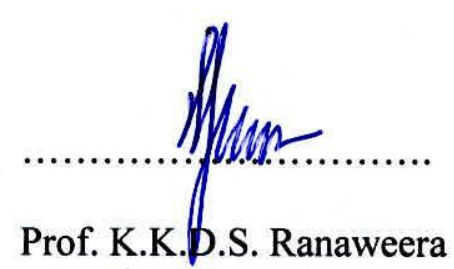

Prof. K.K.D.S. Ranaweera

Head/Department of Food Science and Technology,

Faculty of Applied Sciences,

iniversity of Sri Jayewardenepura,

Gangodawila,

Nugegoda, Sri Lanka. 
We certify that the candidate has incorporated all corrections, additions and ammendments recommended by the examiner.

\section{D.........eypofralua}

Mrs. Damitha Rajapaksa

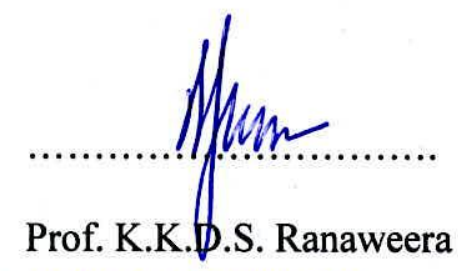

Prof. K.K.D.S. Ranaweera Head/Department of Foodscience and Technology. Faculty of Applled Sciences, University of Sri Jayewardenepura, Gangodawila,

Nugegoda, Sri Lanka.

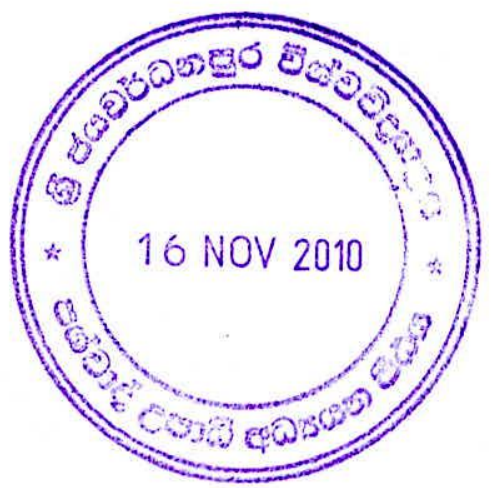





\section{TABLE OF CONTENTS}

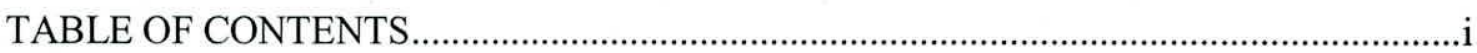

LIST OF TABLES ............................................................................................. vii

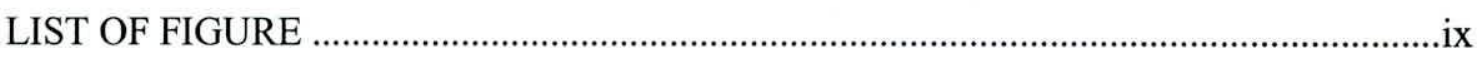

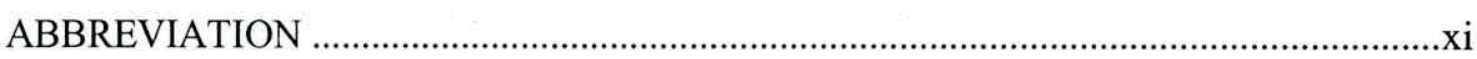

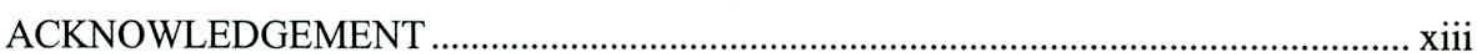

ABSTRACT

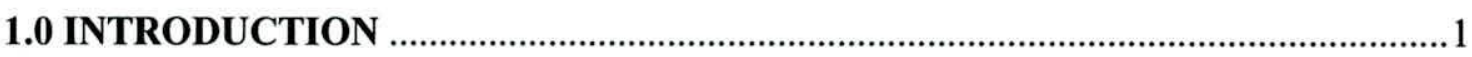

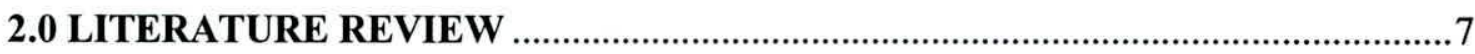

2.1 Rice overview...................................................................................................

2.2 Structure of rice grain ...................................................................................

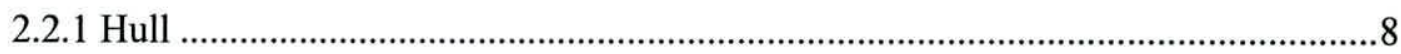

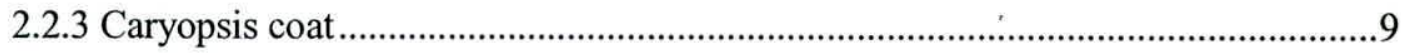

2.2.4 Aleurone layer.........................................................................................

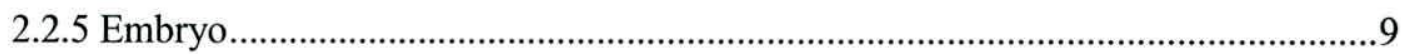

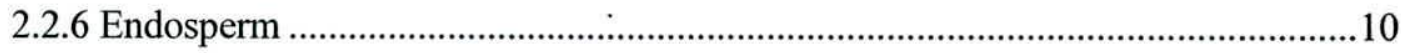

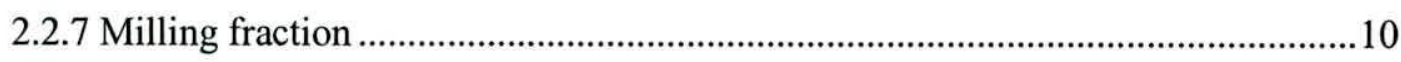

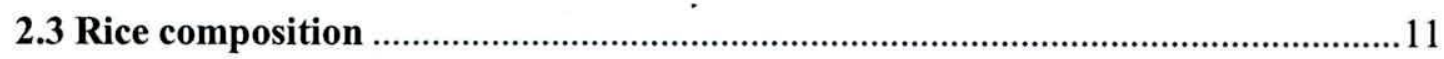

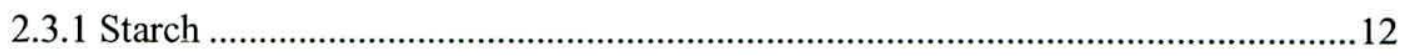

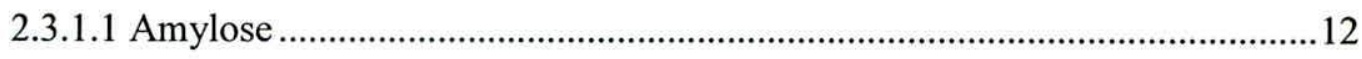

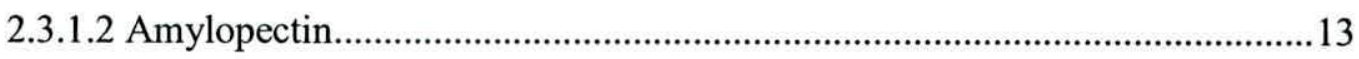

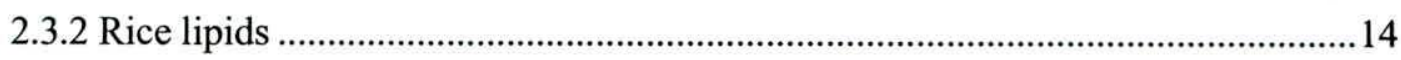

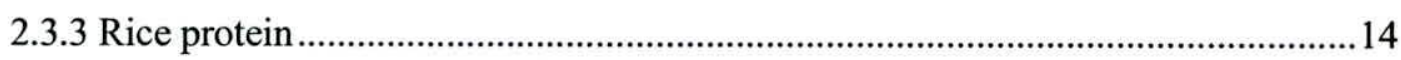

2.4 Functional properties of rice..........................................................................

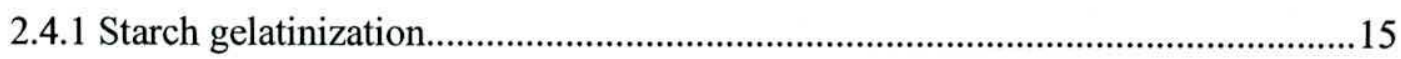

2.4.2 Retrogradation or setback .........................................................................

2.4.3 Pasting behaviour ............................................................................................

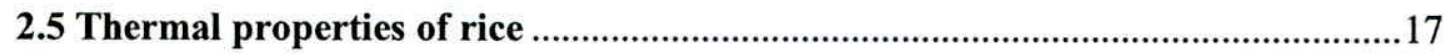

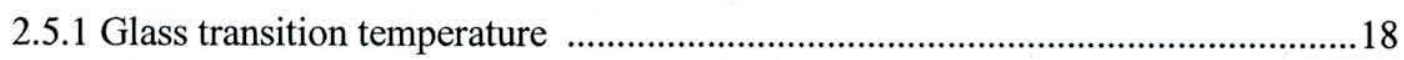




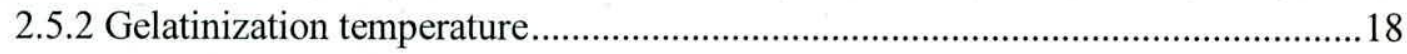

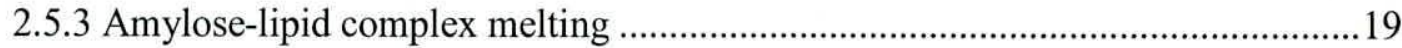

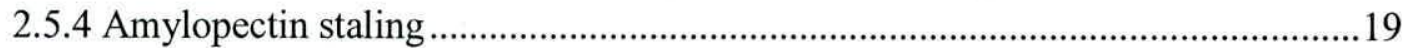

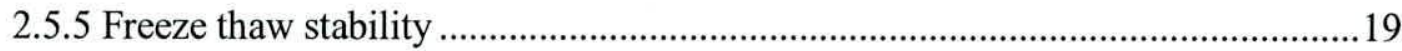

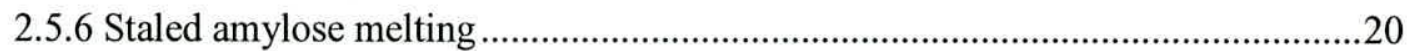

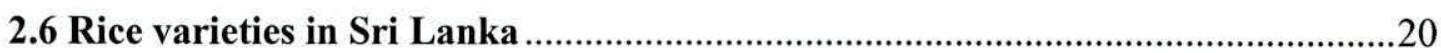

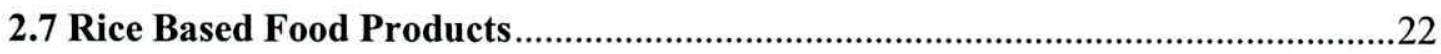

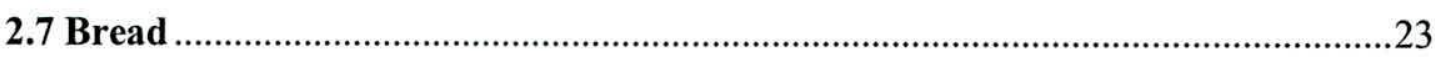

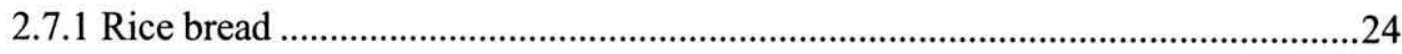

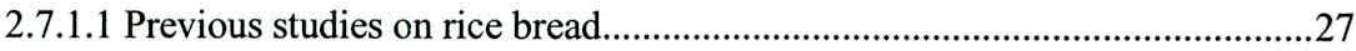

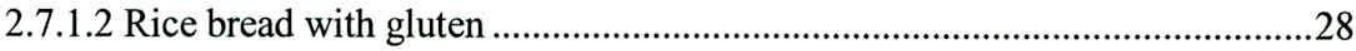

2.7.1.3 Rice bread with hydrocolloids .....................................................................28

2.7.1.4 Varietal influence on rice bread .....................................................................30

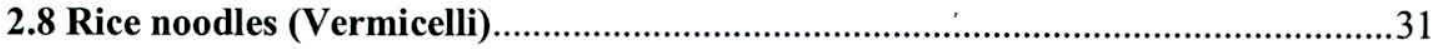

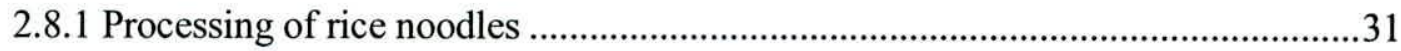

2.8.1.1 Wet milled rice noodles ..................................................................................

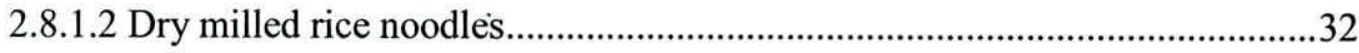

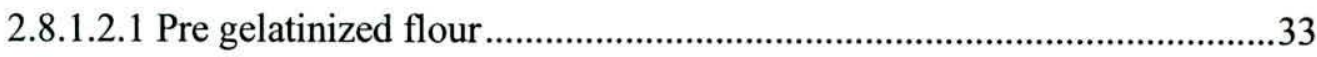

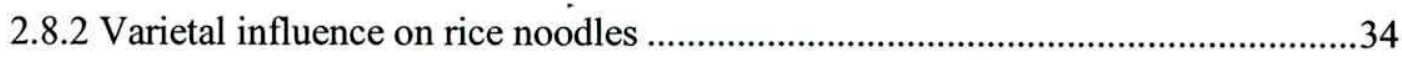

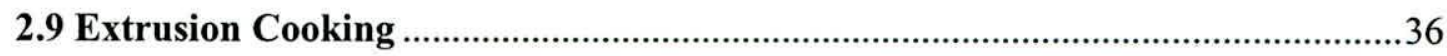

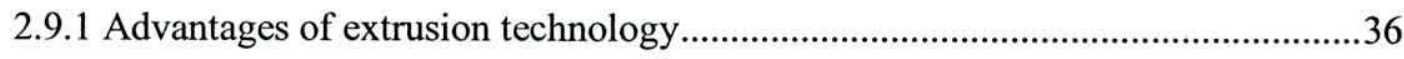

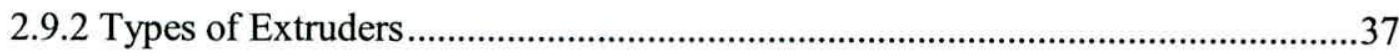

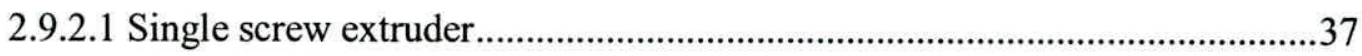

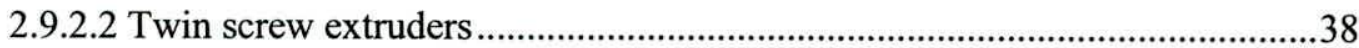

2.9.2.2.1 Counter rotating twin screw extruder ....................................................39

2.9.2.2.2 Co rotating twin screw extruder...............................................................39

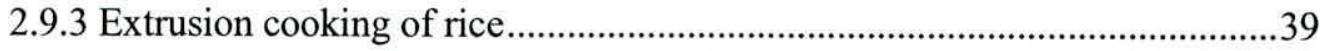

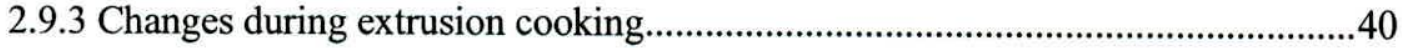

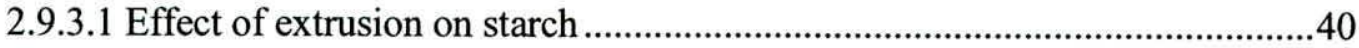

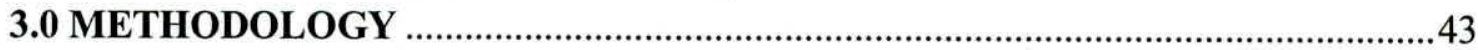


3.2 Preparation of sample

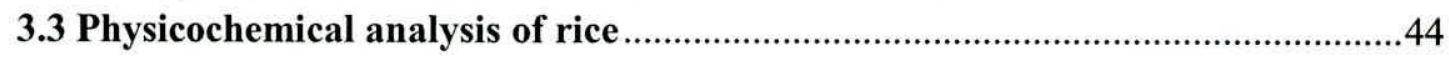

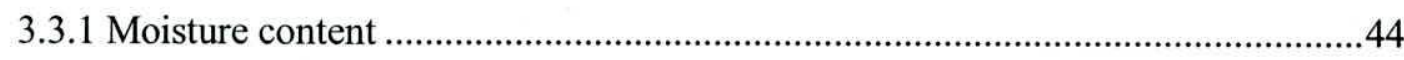

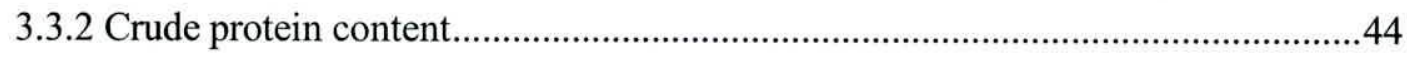

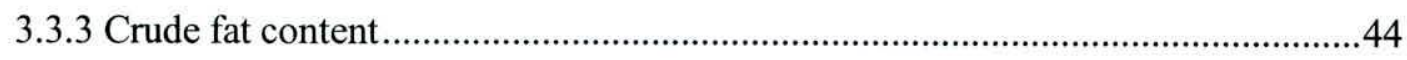

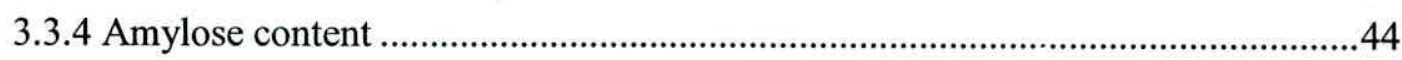

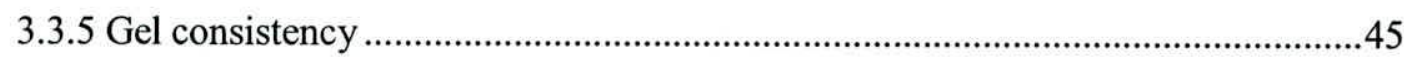

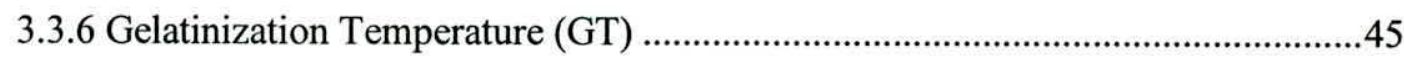

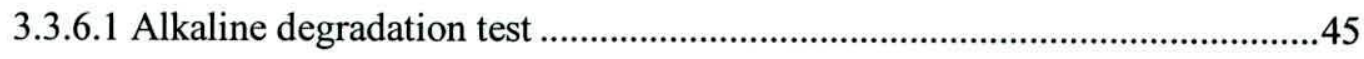

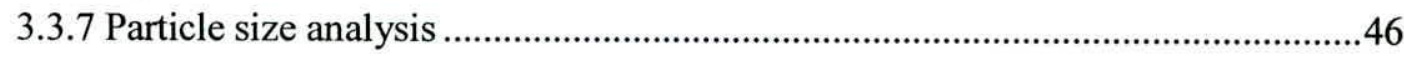

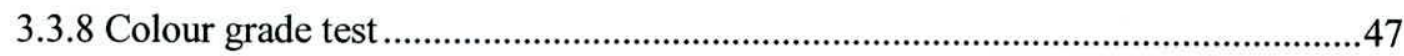

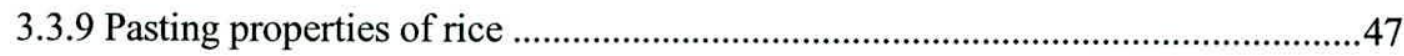

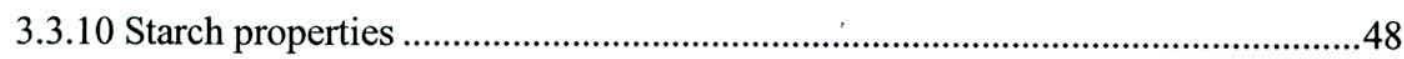

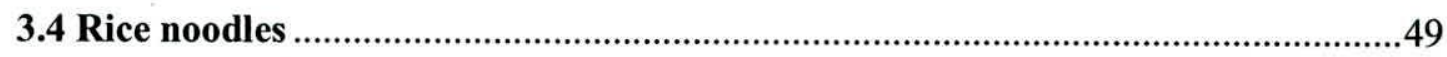

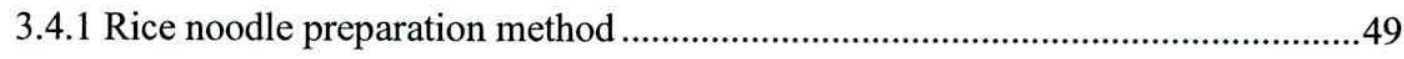

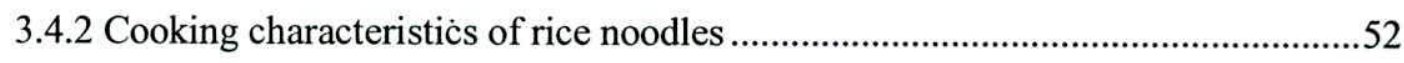

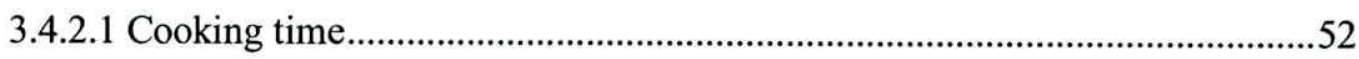

3.4.2.2 Cooking loss or Total solids in gruel .........................................................52

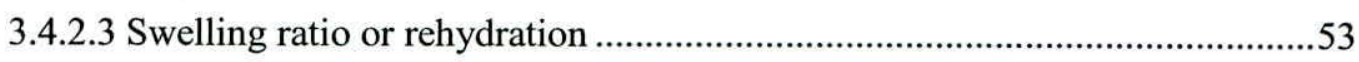

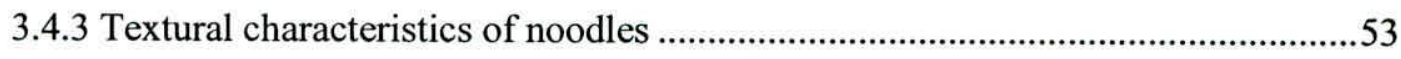

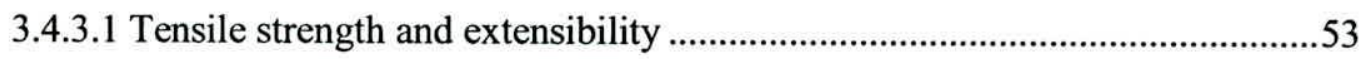

3.4.3.2 Firmness and elastic recovery of noodles .......................................................53

3.4.4 Sensory evaluation of rice noodles .......................................................................5

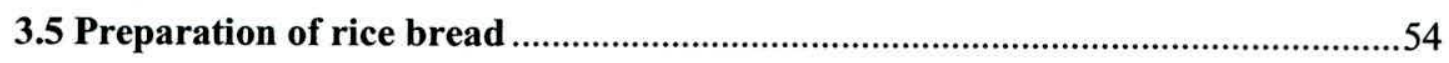

3.5.1 Physical dough properties of dough.......................................................................5

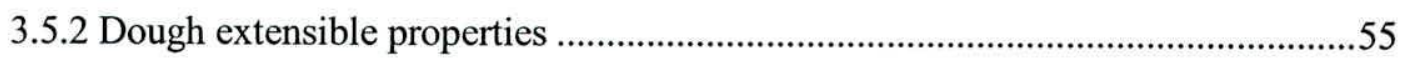

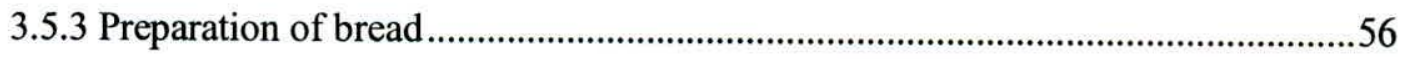

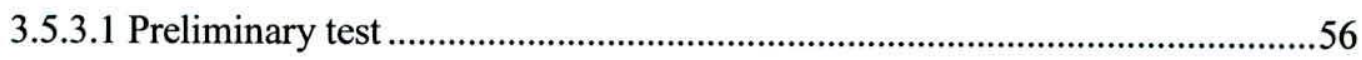

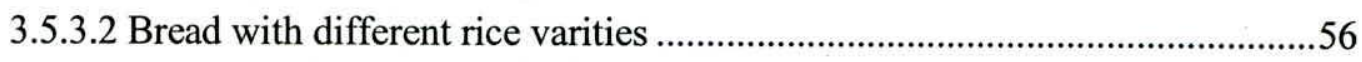




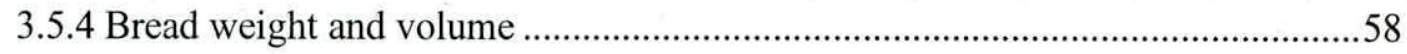

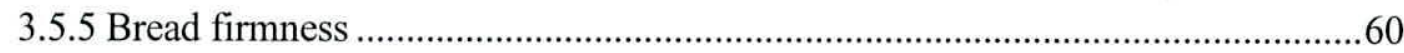

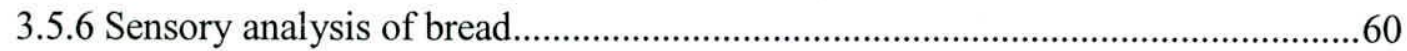

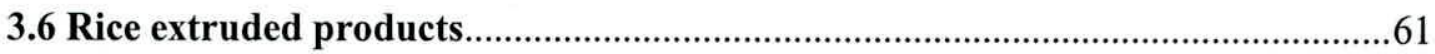

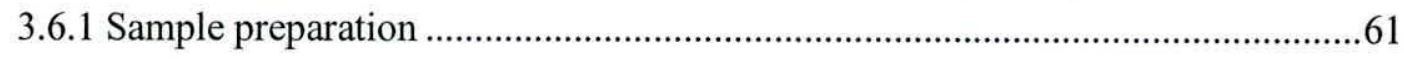

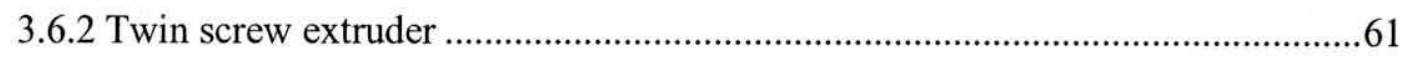

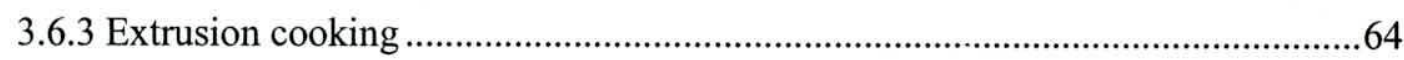

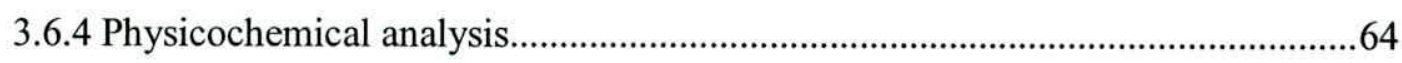

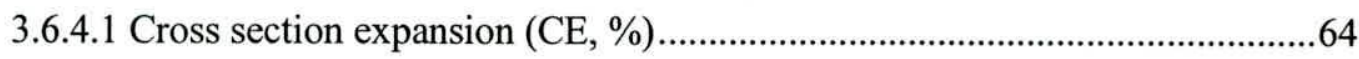

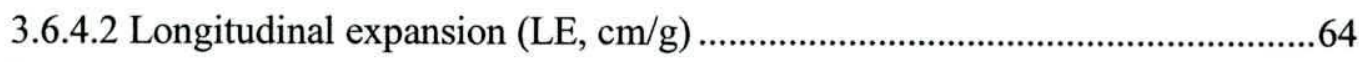

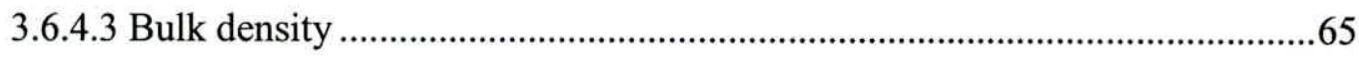

3.6.4.4 Water Solubility Index (WSI), Water absorption index (WAI)....................65

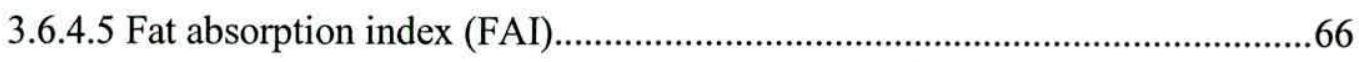

3.6.4.6 Shear Strength or Hardness.........................................................................66

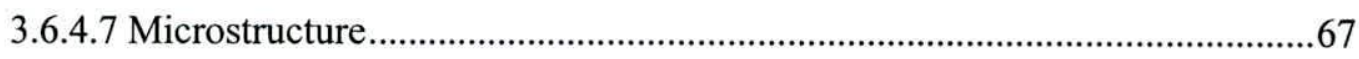

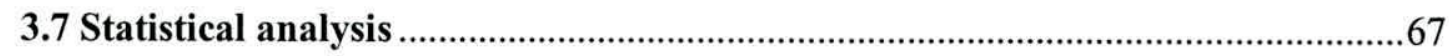

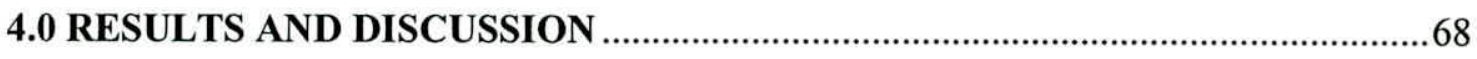

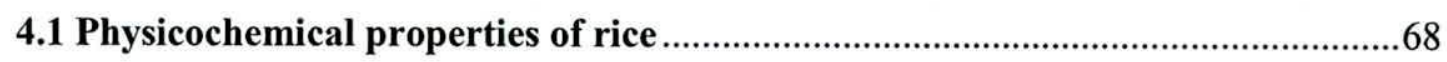

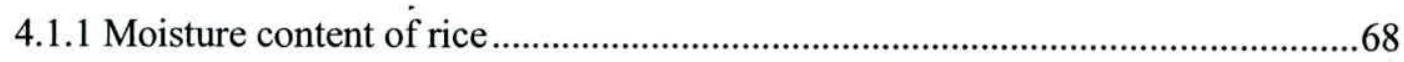

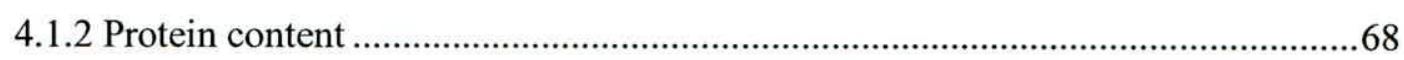

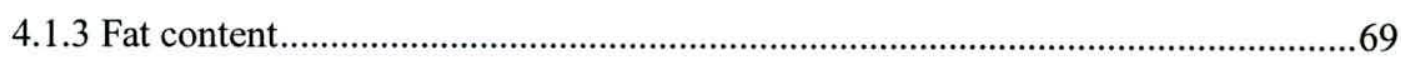

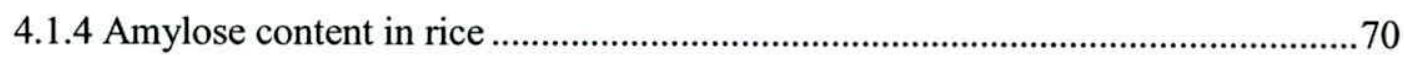

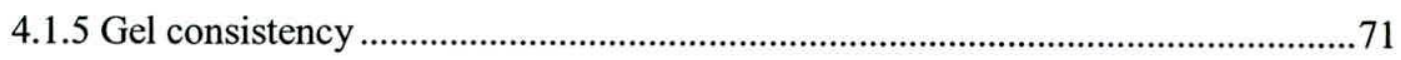

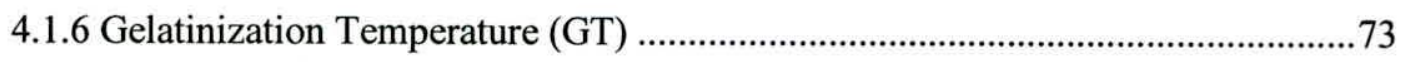

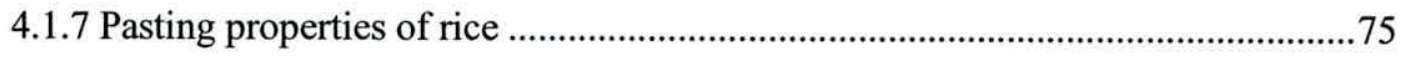

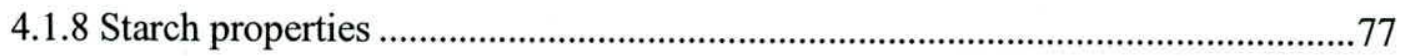

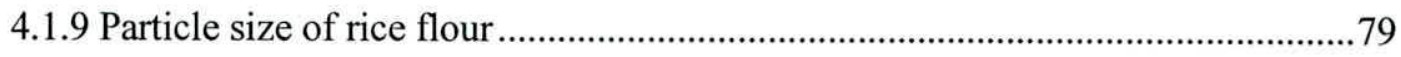

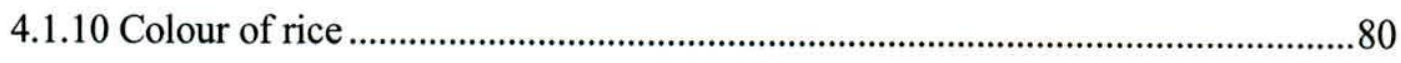

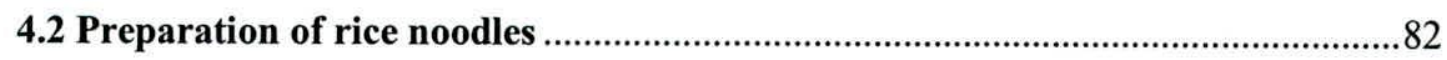

4.2.1 Rice noodles from different rice varieties.........................................................8 82 


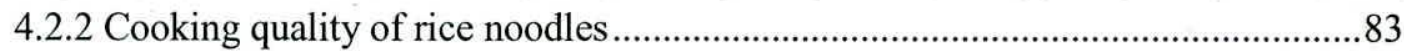

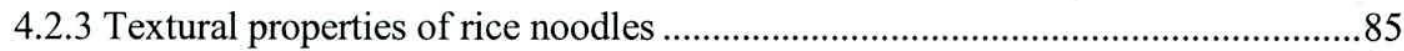

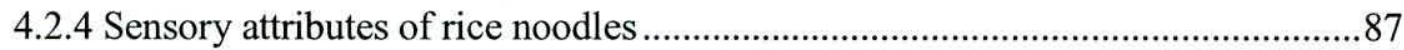

4.2.5 Relationship between physicochemical properties of rice and noodle quality

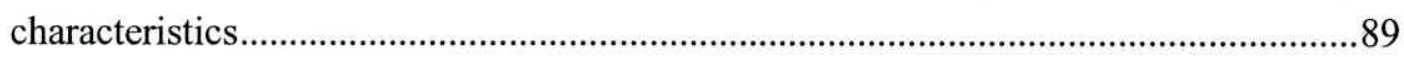

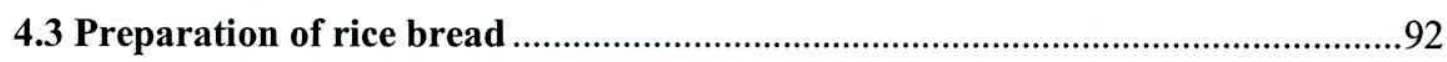

4.3.1 Dough rheological properties of composite mixture with $30 \%$ of rice flour.......92

4.3.1.1 Water absorption capacity..............................................................................92

4.3.1.2 Dough development time ............................................................................93

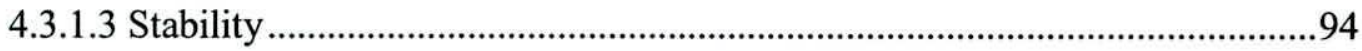

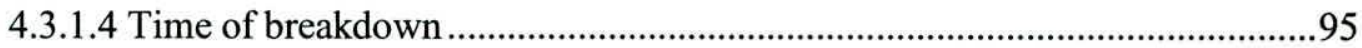

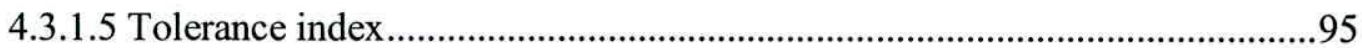

4.3.2 Extensograph measurements of composite flour mixture with $30 \%$ rice flour ...95

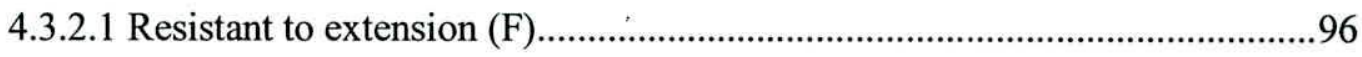

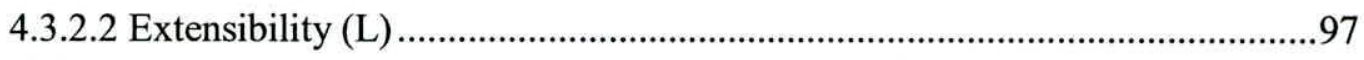

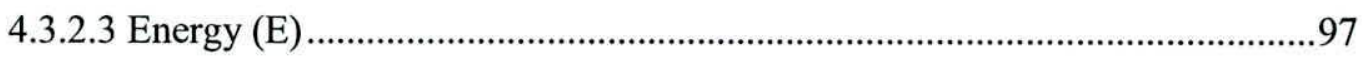

4.3.2.4 Ratio between resistance to extension and extensibility $(\mathrm{R})$.......................98

4.3.2.5 Extensograph properties with different fermentation time............................98

4.3.3 Properties of bread loaves prepared in the preliminary trial..............................100

4.3.4 Bread properties of composite flour mixture with $30 \%$ rice flour......................100

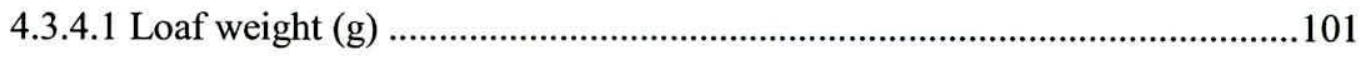

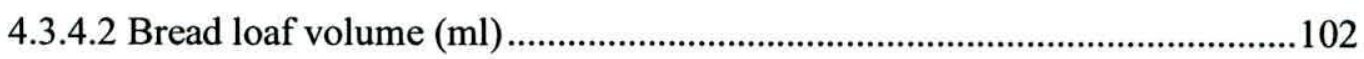

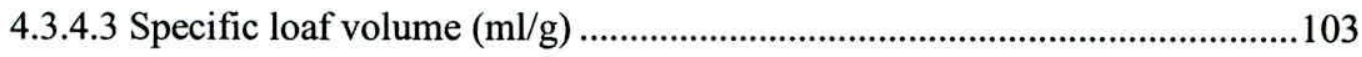

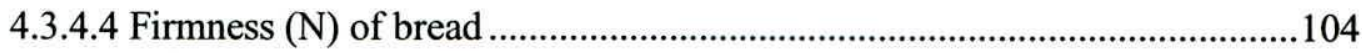

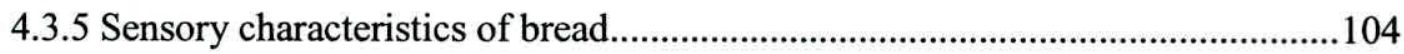

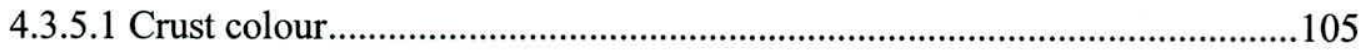

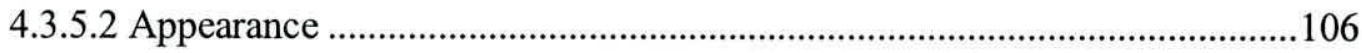

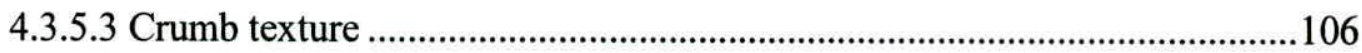

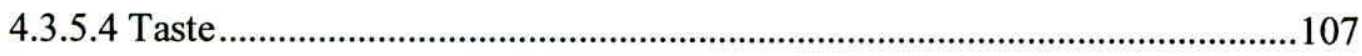

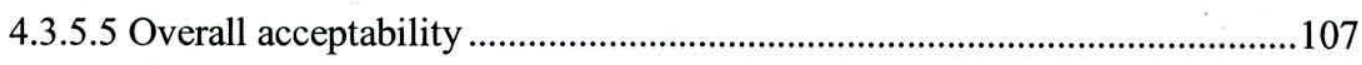


4.3.7 Relationship of physicochemical properties of rice and Farinograph properties with bread properties.

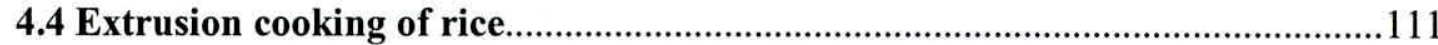

4.4.1 Rice extrudates from different rice varieties...................................................111

4.4.2 Properties of extrudates made from different rice varieties................................112

4.2.2.1 Expansion of rice extrudates .......................................................................112

4.4.2.2 Bulk Density of rice extrudates..................................................................113

4.2.2.3 Water Solubility Index (WSI) and Water absorption index (WAI) of rice extrudates

4.2.2.4 Fat absorption index (FAI) of rice extrudates ............................................118

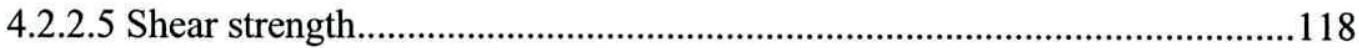

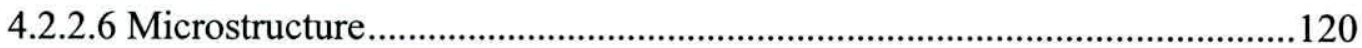

4.3.3 Relationship between physicochemical characteristics of and extruded properties

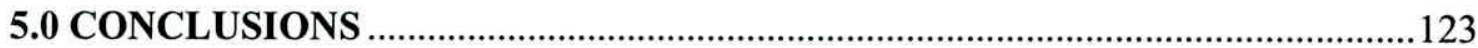

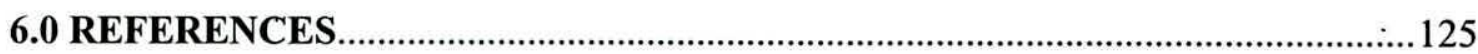

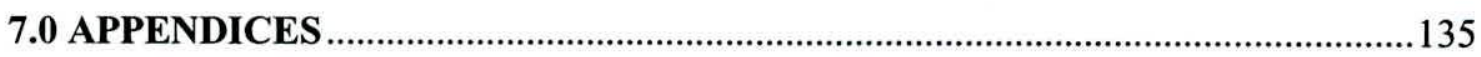

Appendix 1 - List of Publication \& Communication ......................................................135

Appendix 2 - Detailed information on rice varieties studied ...........................................136

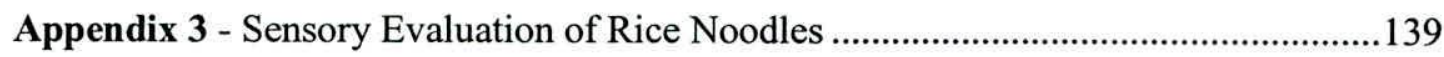

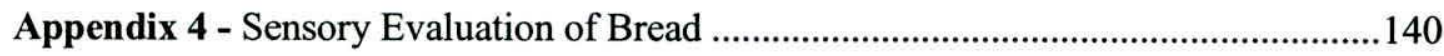

Appendix 5 - Schematic diagram of Amylograph ..........................................................141

Appendix 6 - Farinograph obtained from different rice varieties incorporated at $30 \%$ level dough............................................................ 


\section{LIST OF TABLES}

Table 2.1 Gross composition of rice fractions

Table 2.2 New improved rice varieties recommended by the Department of Agriculture,

Sri Lanka

Table 2.3 Types of protein in wheat and rice (\% of total protein)

Table 3.1 Description of alkali spreading value for milled rice, after soaking in $1.7 \%$ $\mathrm{KOH}$ for 23 hours.

Table 3.2 The screw configuration of the twin-screw extruder

Table 4.1 Protein and fat content of rice

Table 4.2 Amylograph pasting properties of rice

Table 4.3 Starch properties of rice

Table 4.4 Particle size distribution of rice flour

Table 4.5 Chromameter indices for different rice varieties

Table 4.6 Textural characteristics of rice noodles

Table 4.7 Sensory attributes of rice noodles

Table 4.8 Correlation between physicochemical properties of rice varieties and noodle quality

Table 4.9 Physical dough properties of composite flour mixture with $30 \%$ rice flour

Table 4.10 Extensograph properties of dough after 45 minutes

Table 4.11 Properties of bread made with different proportion of rice flour

Table 4.12 Physical properties of rice bread

Table 4.13 Sensory characteristics of bread 
Table 4.14 Correlation coefficient of physicochemical and dough properties with bread quality attributes

Table 4.15 Water Solubility Index (WSI) and Water absorption index (WAI) of rice extrudates

Table 4.16 Correlation between physicochemical properties of rice and extrudate properties 


\section{LIST OF FIGURE}

Figure 1.1 Extent of paddy sown and harvested in Sri Lanka

Figure 1.2 Annual production (000' MT) and average yield $(\mathrm{kg} / \mathrm{Ha})$ of paddy in Sri Lanka2

Figure 2.1 Longitudinal section of rice grain $\quad 8$

$\begin{array}{ll}\text { Figure 2.2 Structure of amylose chain } & 13\end{array}$

$\begin{array}{ll}\text { Figure 2.3 Structure of amylopectin chain } & 13\end{array}$

$\begin{array}{lr}\text { Figure 2.4 Processed rice based products } & 22\end{array}$

$\begin{array}{ll}\text { Figure 3.1 Brabender Amylograph } & 48\end{array}$

Figure 3.2 Flow chart of rice noodles processing $\quad 50$

Figure 3.3 Schematic diagrams of rice noodles processing 51

Figure 3.4 Flow chart of rice bread processing 57

$\begin{array}{lll}\text { Figure 3.5 Processing steps of rice bread } & \cdot & 58\end{array}$

Figure 3.6 Longitudinal view of twin-screw extruder $\quad 62$

$\begin{array}{ll}\text { Figure 3.7 Frontal view of extruder } & 62\end{array}$

$\begin{array}{lr}\text { Figure 4.1 Moisture content of rice } & 68\end{array}$

$\begin{array}{ll}\text { Figure 4.2 Amylose content in brown rice and polished rice } & 70\end{array}$

Figure 4.3 Gel length of rice and their corresponding GC classification $\quad 72$

Figure 4.4 Alkali spreading value of rice in $1.7 \% \mathrm{KOH}$ and their corresponding GT

$\begin{array}{ll}\text { classification } & 74\end{array}$

Figure 4.5 Rice noodles made from different rice varieties 82

Figure 4.6 Cooking quality of rice noodles $\quad 84$ 
Figure 4.8 Extensibility of dough at different times of fermentation

Figure 4.9 Energy of dough at different times of fermentation

Figure 4.10 Ratio of dough at different times of fermentation

Figure 4.11 Firmness of rice bread

Figure 4.12 Bread prepared from 30\% rice flour incorporated with different rice varieties

Figure 4.13 Rice extrudates from different rice varieties

Figure 4.14 Cross sectional expansion (CSE) and Longitudinal expansion (LE) of rice extrudates

Figure 4.15 Bulk densities of rice extrudates from different rice varieties

Figure 4.16 Fat absorption index of rice extrudates

Figure 4.17 Shear strength values of rice extrudates

Figure 4.17 Cell number per cross sectional area of extrudates 


\section{ABBREVIATION}

${ }^{\circ} \mathrm{C}$ - Degree Celsius

$\mu \mathrm{m}$ - micro meter

$\mathrm{a}^{*-}$ red-green colour

AACC - American Association of Cereal Chemists

AC-Amylose content

AOAC - Association of Official Analytical Chemists

AP - Amylopectin

At - Ambalantota

$\mathrm{b}^{*}$ - yellow-blue colour

$\mathrm{Bg}$ - Bathalagoda

BU - Brabender units

Bw - Bombuwala

CE - Cross-sectional expansion

CIE - International Commission on Illumination

$\mathrm{CMC}$ - carboxymethylcellulose

Da-Dalton

DDT - Dough development time

DOM - Degree of milling

DPn - Degree of polymerization

E - Energy

ER (\%) - Elastic recovery

F - Resistant to extension 
F (\%) - Firmness

$\mathrm{GC}-\mathrm{Gel}$ consistency

GO - glucose oxidase

GT - Gelatinization Temperature

HPMC - Hydroxypropyl methyl cellulose

HRW - Hard red winter

HTST - High temperature short temperature

IRRI - International Rice Research Institute

L - Extensibility

$\mathrm{L}^{*}$ - Brightness

Ld - Labuduwa

LE - Longitudinal expansion

MC - Methylcellulose

OIA - Oil Absorption index

PS 50-Median particle size

RMS - Raw Material Specification

RVA - Rapid Visco Analyzer

SLSI - Sri Lanka Standards Institution

SLV - Specific loaf volume

$\mathrm{Tg}-$ Glass transition temperature

Tm - Enthalpy of glass melting endotherm

WAI - Water absorption index

WSI - Water solubility Index 


\section{ACKNOWLEDGEMENT}

I would like to express my heartfelt thanks to my supervisor Mrs. Damitha Rajapakse, Senior Research Officer, Food Technology Section, Industrial Technology Institute (ITI) for her invaluable advises, persistent and generous help and highly encouraging guidance extended to conduct this study. Her unhesitant help and co-operation at all times has contributed immensely to make this study a success.

I wish to express my sincere appreciation and gratitude to my supervisor, Prof. K.K.D.S. Ranaweera, Head, Department of Food Science \& Technology, University of Sri Jayewardenapura, for his valuable advice, kind guidance and professional expertise throughout my study.

My deep sense of gratitude is expressed to Dr. (Ms.) Janaki Gooneratne, Head, Food Technology Section, Dr. (Ms.) Shanthi Wilson, Deputy Director - Research \& Development, ITI, and Dr. A.M. Mubarak, Director, ITI for granting me the opportunity to carry out this study.

I am deeply grateful to Mrs. Theja Herath, SRO, Mr. Nuwan Ranaweera, RO, Mr. Tharaka Gunawardena, RO, and Mr. D.M. Weerawardena for supporting me with my work in many ways. The secretarial assistance of Mrs. Lilani Gunathilaka, Mrs. Prasadi Sapukotuna and Ms. Udari Weerasingha are gratefully acknowledged. 
I also express my gratitude to Mrs. Karuna Aponso, TO, Mrs. R.K.P.N. Indunil TA, Mrs. Agnes Fernando, STO, Mrs. Shiranthi Perera, TO and all the staff members of Food Technology Section who helped in numerous ways during the period of this study.

I wish to acknowledge Dr. Amitha Benthota, Senior Research Officer and Dr. Gamini Dissanayaka, Director, Rice Research and Development Institute, Bathalagoda for providing me the rice samples and necessary information for my studies.

I extend my thanks to Dr. (Mrs.) Nandani Ediriweera, Consultant Food Technology, 2KR Project, Mrs. Dilamani Warnasuriya, Head, Information Technology Centre, ITI and Mrs. Purnima Jayasinhgea Information Technology Centre, ITI of for their fullest support during finalizing of the thesis. I extend my thanks to Mr. Gamini Deshapriya, Laboratory assistant for his kínd help in various ways.

Also I wish to thank all the staff members of ITI who helped in many ways to furnish this study successfully specially the staff members of Information Service Centre, ITI.

Finally I extend my gratitude to my ever loving parents and wife, who all way behind me to bring this as success. 


\section{SELECTION OF RICE VARIETIES SUITABLE FOR RICE BASED FOOD PRODUCTS}

M.J.M. Fari

\section{ABSTRACT}

Rice (Oryza sativa L.) is the staple food of Sri Lanka and achieved self sufficiency in rice production. Diversification of rice based product will enable the consumer to have a wide range of products. A wide range of rice varieties are being cultivated under different agroclimatic conditions in Sri Lanka. These different varieties having compositional differences, contribute to the diversity of physicochemical properties. A study was carried out to select the suitable rice varieties for manufacturing rice based food products such as bread, noodles and extrudates.

Popularly grown Sri Lankan rice varieties namely Bg 300, Bg 352, Bg 403, Bg 94-1, Ld 356, Bw 272-6b, At 405 and At 306 were used for the present study. Physicochemical properties of the rice varieties and the quality characteristics of rice noodles, rice bread and rice extrudates were investigated.

Physicochemical properties of rice had shown a wide variation among different rice varieties. Amylose content (AC) was high in polished rice than brown rice irrespective of the variety. $\mathrm{AC}$ of polished rice ranged from $21.49 \pm 1.468$ in At 405 to $36.93 \pm 0.346 \%$ in Bg 94-1. Amylograph pasting properties of rice varieties showed a significant $(p<0.05)$ 
variation for all the pasting parameters. Protein content was ranged from $6.84 \pm 0.199$ in $\mathrm{Bg}$ 94-1 to $11.18 \pm 0.219$ in Ld 356.

Rice noodles were prepared by pre gelatinizing the dough followed by cold extrusion.

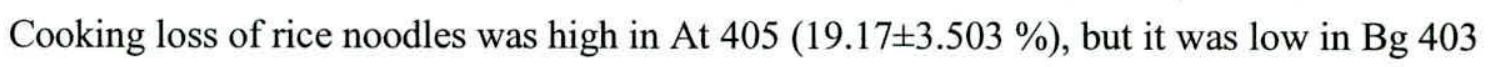
$(9.19 \pm 0.327 \%)$. Tensile strength of noodles was significantly $(\mathrm{p}<0.05)$ high in $\mathrm{Bg} 352$ $(16.7 \pm 3.37 \mathrm{~g})$ whereas it was significantly $(\mathrm{p}<0.05)$ low in At $405(8.0 \pm 1.72 \mathrm{~g})$. Overall acceptability of rice noodles from At 405 had the significantly $(\mathrm{p}<0.05)$ lowest score and rice noodles from $\mathrm{Bg} 300$ had significantly $(\mathrm{p}<0.05)$ higher value. AC had significant correlation with cooking loss, swelling ratio, tensile strength, extensibility and elastic recovery at $\mathrm{p}<0.05$.

Incorporation of different rice varieties at $30 \%$ level into wheat flour had a significant $(\mathrm{p}<0.05)$ variation in physical dough properties, physical bread properties and sensory attributes of bread. Rice variety Bg 352 incorporated bread had the significantly $(\mathrm{p}<0.05)$ highest specific loaf volume $(5.22 \pm 0.108 \mathrm{~g} / \mathrm{ml})$ and lowest firmness $(6.52 \pm 0.299 \mathrm{~N})$.

Rice extrudates were prepared in co-rotating, fully intermeshing twin screw extruders. Wide variation in extrudate properties were observed among rice varieties. Rice variety $\mathrm{Bg}$ 352 and $\mathrm{Bg} 300$ had greater puffing during extrusion. Rice varieties from Bathalagoda exhibit low WSI and high WAI. AC significantly correlated negatively with density (r=$0.365, \mathrm{p}<0.01)$, WSI $(\mathrm{r}=-0.522, \mathrm{p}<0.001)$ and FAI $(\mathrm{r}=-0.352, \mathrm{p}<0.05)$ and positively with 
WAI $(r=0.569, \mathrm{p}<0.001)$. Protein content had significant effect on WSI and WAI. Amylograph pasting properties also had a significant effect on rice extrudate properties.

The amylose content was the major factor affecting the quality characteristics of rice based products, even though the Amylograph pasting properties showed significant differences Two wide grown rice varieties, namely $\mathrm{Bg} 300$ and $\mathrm{Bg} 352$ showed overall best performance for making rice noodles, rice bread and rice extrudates. Also other rice varieties, namely Bg 94-1, Bg 403 and At 403 also preferred in making such products. 